Journal of Clinical and Experimental Neuropsychology, 16, 589-596, 1994.

\title{
Memory for Faces in Epileptic Children Before and After Brain Surgery
}

Elizabeth D. Beardsworth and Dahlia W. Zaidel

Department of Neurology Department of Psychology,

Radcliffe Infirmary, Oxford University of California at Los Angeles

\section{Running head: MEMORY FOR FACES}

Correspondence to: Dr. D. W. Zaidel, Dept. of Psychology, UCLA, 405 Hilgard Ave., Los Angeles, CA 90024-1563, USA

Email: dahliaz@ucla.edu

\begin{abstract}
Memory for pairs of unfamiliar children's faces was investigated in 29 children and adolescents suffering from left (LTLE) or right (RTLE) temporal-lobe epilepsy, before and after temporal-lobe surgery. Both immediate and delayed memory were tested. Before surgery, RTLE subjects performed worse than either LTLE subjects or normal children. After surgery, RTLE subjects improved significantly. Overall (after surgery), there was no significant LTLE-RTLE difference, but on delayed memory, the RTLE group was worse than the LTLE group. The results suggest specialization for facial memory in the right hemisphere of young patients, as in adults, despite early brain damage.
\end{abstract}




\section{INTRODUCTION}

The role of right mesial temporal-lobe regions in facial processing and recall has been consistently demonstrated in adult patients who underwent right anterior temporal-lobectomy for alleviation of epilepsy (Milner, 1958, 1968). Adult patients with right excisions were significantly impaired in recognizing unfamiliar faces as compared to patients with excisions on the left side. Due to the paucity of studies it is not known whether or not younger patients are similarly affected by unilateral brain damage in the temporal-lobe or in other regions.

Memory for faces in children is somewhat different from that of adults. Normal children under 10 years of age performed at a level similar to that of right brain-damaged patients on a clinical test that measures memory for adult faces (Benton \& Van Allen, 1973). Carey and colleagues (Carey, Diamond, \& Woods, 1980) have studied systematically the developmental changes in children's facial memory and have found that 6- to 16- year-old children perform worse than adults. Moreover, children's memory for faces is more sensitive than adults' to facial expression, facial perspective, clothing, or hairstyle (Carey \& Diamond, 1977; Ellis \& Flin, 1990; Ellis, 1992). Carey (1992) has proposed that, unlike adults, children cannot abstract the invariant details of faces nor distinguish distinct features in faces. In view of differences between children and adults, it is important to determine, for theoretical as well as for clinical reasons, the effects of unilateral brain damage (due to epilepsy and brain surgery) on a facial memory test in children. The study reported here is seen as an important first step in this direction.

Clinically, little is known about the specific effects of localized right hemisphere damage in children on nonverbal functions, including face recognition. While much attention is given by both teachers and parents to impairments in left hemisphere skills such as reading and writing, impairments in right hemisphere skills such as facial identification or expression are little studied (Rudel, 1985). This is due, in part, to the ease with which disturbances in language can be recognized, and partly to the availability of theoretical frameworks for language. Yet, in everyday life, facial processing is a crucial component of communication for children as well as for adults and, some would argue, no less important than reading or writing. For instance, developmental 
prosopagnosia, the congenital inability to recognize people by their faces alone, typically goes unnoticed in children and only comes to the attention of neurologists and neuropsychologists when sufferers reach adulthood (Campbell, 1992; McConachie, 1977). Until the present investigation, no clinical test has been devised specifically to test memory for faces in children suffering from brain damage. Any tests that are available use adult faces as stimuli and apply testing procedures that are valid for adult patients. In the test developed for this study, on the other hand, the stimuli are children's faces and the testing procedures are appropriate to them.

The findings for right anterior temporal lobectomy patients should be interpreted against a background of relevant findings on right hemisphere specialization in facial processing in patients with other right hemisphere lesions and in normal subjects. Non-temporal-lobe regions, particularly posterior medial regions in the right hemisphere have been found to be involved in facial processing as well. In nonepileptic adult patients suffering from unilateral damage, including stroke and gunshot wounds, impairments in face recognition have been found by Warrington and James (1967), Benton and Van Allen (1968), DeRenzi, Faglioni, and Spinnler (1968), Yin (1970), Tzavaras, Hecaen, and Le Bras (1970), and Newcombe, de Haan, Ross, and Young (1989). Together, these findings on brain-damaged patients demonstrate that systems within the right hemisphere play a significant role in adult facial processing, both in perception of and memory for familiar and unfamiliar faces.

A left visual half-field, right hemisphere advantage for processing facial information has been demonstrated by de Schonen and Mathivet (1989) in normal infants as young as 4 - 9 months of age and this hemispheric advantage extends to children of primary school age (Ellis, 1990; Levine, 1985; Turkewitz \& Ross-Kossak, 1984). Thus, laterality in facial processing is present quite early in development. Carey (1981) proposed that right hemisphere mechanisms responsible for facial processing (posterior cortex) become temporarily nonfunctional, between the ages of 10 and 14 years, possibly due to effects of hormonal changes. Recently, de Schonen (1992) proposed that facial processing may have a large interhemispheric communication component. 
In assessing the effects of epilepsy or of temporal-lobe surgery on memory for faces in either children or adults, several specific theoretical and clinical questions arise. Does early onset of epilepsy change lateralization in facial processing? What effect does early surgical intervention have on lateralized deficits? Previously, these questions have not been studied systematically and an attempt is made to address them here. Given the foregoing, worse overall performance by the RTLE group was predicted compared to that of the LTLE group and normal children. Considering the well-established therapeutic benefits of temporal lobectomy, we predicted an improvement in performance after surgery.

\section{METHOD}

\section{$\underline{\text { Subjects }}$}

All English-speaking children operated for temporal-lobe epilepsy in the Radcliffe Infirmary, Oxford, between 1983 and 1989 who obtained a WISC-R (Wechsler, 1974) Full Scale IQ of at least 70 before surgery were included in the study $(\underline{\mathrm{N}}=29)$. Preoperative testing took place 3 months before surgery and postoperative testing took place 6 months after surgery. However, not all patients were administered the face test both before and after surgery. Two groups were formed, left temporal epileptic focus (LTLE) and right temporal epileptic focus (RTLE). They all suffered from pharmacologically intractable medial temporal-lobe epilepsy that can be alleviated by anterior temporal-lobectomy. Thirteen ( 8 girls, 5 boys) underwent left-sided surgery and 16 (10 girls, 6 boys) underwent right-sided surgery.

Twenty-seven patients underwent en bloc unilateral anterior temporal-lobectomy (TL) resections (12 left and 15 right) and 2 underwent unilateral selective amygdalo-hippocampectomy (SAH) (1 left and 1 right). TL consisted of resection of the anterior temporal-lobe (approximately $5-5.5 \mathrm{~cm}$ on the left and $5.5-6 \mathrm{~cm}$ on the right), the amygdala, and the anterior $3 \mathrm{~cm}$ of the hippocampus (approximately two-thirds) on either side (Adams, 1987; Oxbury \& Oxbury, 1989). The SAH surgery consisted of removal of the amygdala and the anterior $3 \mathrm{~cm}$ of the hippocampus (approximately two-thirds). The surgical approach in SAH consisted of an incision through the middle temporal gyrus (Oxbury \& Adams, 1989) rather than through the Sylvian fissure. The basis 
for selection of patients to undergo the surgical procedure was the same regardless of which side was involved, namely, detailed medical history, drug history, neurological examination, electroencephalography, brain imaging including contrast studies, neuropsychological evaluation, and, whenever appropriate, carotid Amytal testing procedure. In addition, the patients had to have suffered from seizures despite therapeutic levels of medication for at least 2 years prior to surgery. Clinically, the postoperative course at the time of testing was good and the same in both patient groups.

There were no statistically significant left-right differences in Full Scale IQs (Table 1) nor in the Verbal or Performance Scales (although the trend was in the direction of lower Verbal Scale scores in the LTLE group). Most patients were right-handed, except for two children. Language dominance was established by carotid Amytal testing and neuropsychological evaluation. Only in 12-year-old or older patients was the carotid Amytal procedure applied. Twenty-five patients had left hemisphere dominance for language as determined by the Amytal procedure; 4 had "mixed" language dominance (2 LTLE and 2 RTLE) as determined by this procedure. In the "mixed" criterion, patients were judged to have "greater" left hemisphere language dominance rather than equal language representation bilaterally.

The age of onset of the epilepsy in the two groups was not statistically different. In the majority of cases, the age of onset dated back to early childhood and the etiology in most was hippocampal sclerosis. In some cases indolent glioma was present, presumably since birth, and was considered the cause of the epilepsy. None suffered from head trauma. The first seizure in most children occurred prior to age 4 . Table 1 provides a summary of the clinical histories.

Eighteen normal children (9 girls, and 9 boys) free from neurological disorders were recruited from a local Oxfordshire school. Their mean age was 12 years, 6 months (range 12 - 13 years) and all obtained a Full Scale IQ score of at least 70 on the WISC-R.

\section{$\underline{\text { Materials }}$}

The Beardsworth Memory for Children's Faces Test (BMCFT) was conceived and devised by one of us (EDB). It consists of $4 \times 3$ in. black and white photographs of normal, unfamiliar children's 
faces. They are all straight-on front views and professionally photographed. The photographs represent equally boys and girls who range in age from 8 to 12 years. Two sets of faces, Set I and Set II, with 10 pairs in each were prepared. A pair consists of a boy and a girl. One set was administered before surgery and the other set was administered after surgery.

Design and Procedure

Each of the sets was administered on six separate trials in the following manner: There were five consecutive immediate memory trials and one delayed memory trial (the sixth). Each trial consisted of the following procedure: Ten pairs of photos (of a boy and of a girl) were laid out on a table in two vertical columns consisting of five boy-girl pairs per column. In all trials, the pairs were the same but their placement within the two columns varied from trial to trial. When the stimuli were positioned on the table, the subject was told that these are pictures of pairs of friends and that he/she has to try to remember which boy goes with which girl. The study period lasted $30 \mathrm{~s}$. When immediate memory was tested, after subjects studied the array, the experimenter removed all photos of girls, leaving on the table a rearranged array (i.e., not in columns anymore) of the photos of boys. The subject was handed the photos of girls, one at a time, to match with the appropriate boy partner on the table ("Find the photo of the friend of the girl in this photo."). Occasionally, subjects corrected themselves and these were allowed. Correct placing were acknowledged by the experimenter. When matches were found for all photos, feedback on incorrect matches was given. When the trial was completed, the photos were removed, and the next trial began in the same manner as described above. In the delayed memory trial, the identical procedure was followed except that a 30-min filled delay separated the end of the study period from the beginning of the test phase. Thus, after viewing the photo array for $30 \mathrm{~s}$, all stimuli were removed and the delay period began.

Whenever possible, patients were tested both before and after surgery, with a different set each time. They were randomly assigned to one or the other set regardless of side of surgery, sex, age, or any other consideration. In the LTLE group, 11 patients had both pre- and postoperative tests; in the RTLE group, 10 patients had both pre- and postoperative tests. 
Normal children were administered Set I first and Set II a few days to a week later (but delayed memory was not tested in Set II). This was done in order to establish that the two sets were equally difficult, since practice effects across sets can be assumed to be negligible. The results showed that there was no difference in performance on Sets I and II (described below).

\section{RESULTS}

Accuracy scores on the BMCFT were tabulated for the normal subjects, the LTLE, and the RTLE groups. Chance performance was a score of $17.5 \%$ correct on each trial. All three groups obtained above-chance mean scores on each of the trials, including the very first one.

In normal children, there was no significant difference between the scores on all five of the immediate memory trials in Set I $($ mean $=50.9 \%)$ and those in Set II (mean $=53.3 \%)$. Absence of substantial difference indicates that the two sets are equivalent. Hence random assignment of patients to be tested on either Set I or Set II was justified. Given lack of significant difference between Set I and II, the average of both sets was used in statistical comparisons between normal and patients groups. The sensitivity of the delayed memory trial was analyzed separately. The difference between Trial 5 in Set I (which was the last of the immediate memory trials) and Trial 6 (delayed memory) was significant $(\underline{F}(1,17)=4.75, \underline{p}<.04)$. It represents the drop from a score of $66.6 \%$ to $59.4 \%$, respectively.

Preoperative data

The logic behind the statistical analyses was to include as many patients as possible, rather than to compare the performance of only those patients who had both pre- and postoperative scores alone. However, the change from pre- to postoperative testing was analyzed only on those with pre- and postoperative scores.

Figure 1 illustrates performance before surgery. The overall mean score on all six trials was $50.9 \%$ for the LTLE group and $35.0 \%$ for the RTLE group. As predicted, the difference between the two groups on all six trials was significant $(\underline{F}(1,19)=4.21, \underline{p}<.03$, one-tailed $)$. Performance on the delayed memory trial was significantly different between the two groups ( $\underline{F}$ (1, $19)=3.82, \underline{p}<.05)$. Immediate memory (collapsed across all five trials) was not significantly 
different between the left and right groups. Similarly, there was no statistical difference between the last of the immediate memory trials (Trial 5) and the delayed memory trial, in either group. Mean scores of the RTLE group were consistently lower than those of the LTLE group. Only the RTLE subjects performed significantly worse $(\underline{F}(1,26)=6.50, \underline{p}<.01)$ than normal on all six trials. And, as predicted, the RTLE group differed significantly from normal on the delayed trial ( $\underline{\mathrm{F}}$ $(1,26)=3.40, \underline{\mathrm{p}}<.03$, one-tailed $)$.

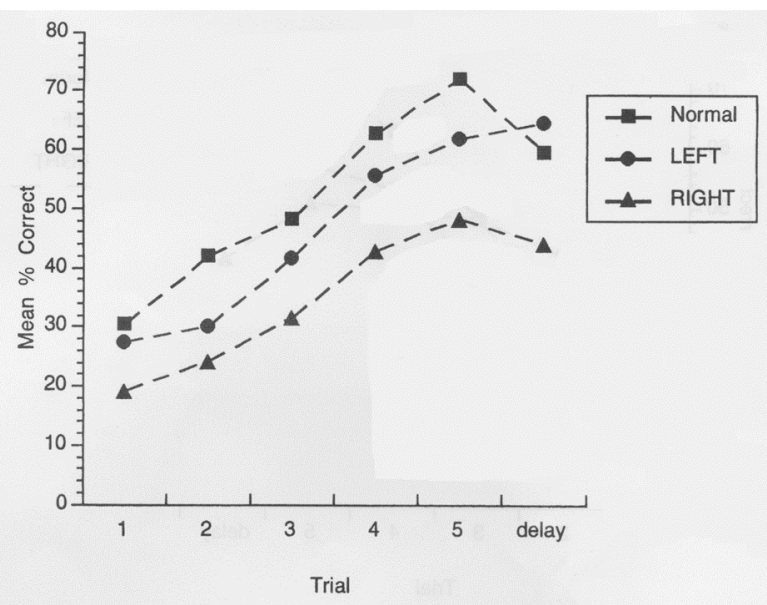

Fig. 1. Mean accuracy scores (\%) of the normal group (average of Sets I and II) and of the LTLE and RTLE groups preoperatively.

\section{$\underline{\text { Postoperative data }}$}

Figure 2 shows patients' performance following surgery. No statistical difference between the two groups for all six trials was found. But, importantly, the difference on the delayed memory trial was statistically significant $(\underline{\mathrm{F}}(1,24)=3.84, \underline{\mathrm{p}}<.03$, one-tailed $)$, as expected given the sensitivity of medial temporal-lobe surgery to delay trials and the normal right hemisphere specialization for facial processing. 


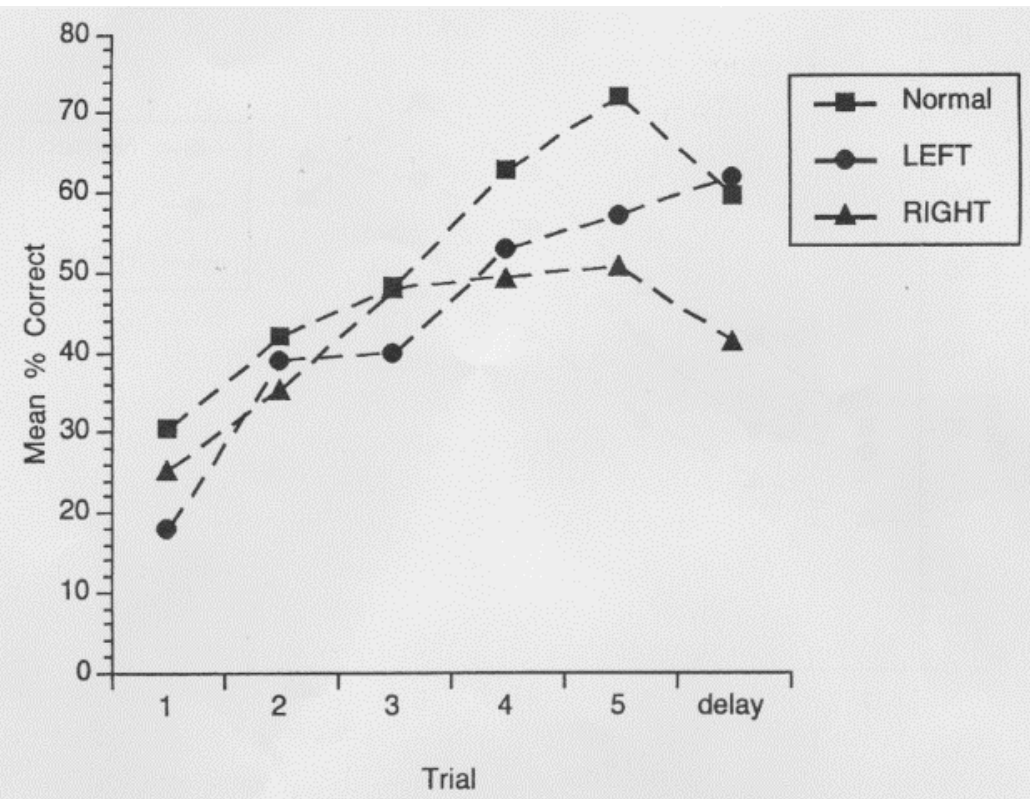

Fig. 2. Mean accuracy scores (\%) of the normal group (average of Sets I and II) and the patient groups postoperatively.

Only in the RTLE group was there a statistical difference between the last immediate memory trial (Trial 5) and the delayed memory trial $(\underline{\mathrm{F}}(1,15)=4.37, \underline{\mathrm{p}}<.05)$. This statistically significant difference was not found in preoperative performance, although the trend was clearly present then. Normal subjects as well (described above) obtained significantly worse scores on the delayed trial than on Trial 5. In the LTLE group the difference was not significant.

\section{$\underline{\text { Preoperative compared to postoperative data }}$}

The scores of the patients to whom both pre- and postoperative tests were administered were analyzed (Figure 3). The RTLE group improved significantly both on immediate $(\underline{\mathrm{t}}(9)=-1.88, \underline{\mathrm{p}}$ $<.04$, one-tailed) and on delayed memory $(\underline{\mathrm{t}}(9)=-2.01, \underline{\mathrm{p}}<.05$, one-tailed while there was no statistical change in the LTLE group. 

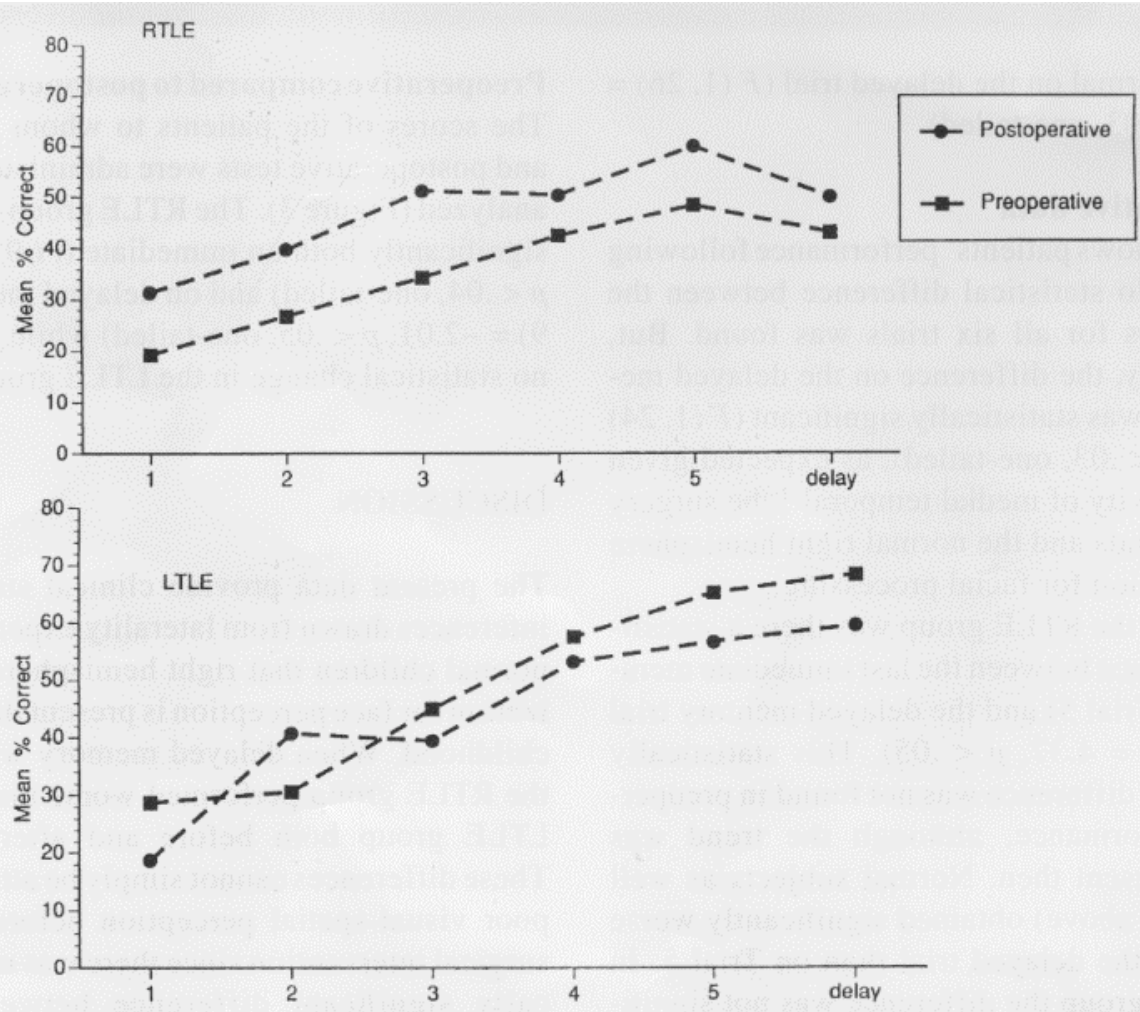

Fig. 3. Performance of matched patients in the LTLE and the RTLE groups. They were administered the facial memory test both before and after surgery.

\section{DISCUSSION}

The present data provide clinical support for inferences drawn from laterality experiments in normal children that right hemisphere specialization for face perception is present throughout childhood. When delayed memory was tested, the RTLE group performed worse than did the LTLE group both before and after surgery. These differences cannot simply be attributed to poor visual-spatial perception before or after surgical intervention since there was no statistically significant difference between these groups on Performance IQ, nor can they be attributed to reduced intellectual functioning since there was no significant difference on the Verbal IQ or Full Scale IQ. The most parsimonious explanation for the observed hemispheric differences is that they are consistent with the predominant role attributed to systems within the right hemisphere in facial processing. 
Surgery had a beneficial effect on performance of the RTLE group. The data show that the preoperative deficits in patients with right temporal-lobe epilepsy are due to epileptic disruption of regions beyond the operatively (to be) excised tissue, and that these deficits improve following surgery. Although it was the RTLE group that obtained lower scores than the LTLE group or normal subjects, it must be stressed that the focal lesion is in the anterior temporal-lobe. As described earlier, other regions within the right hemisphere are involved in facial processing, and normal facial processing could have taken place in those intact regions. The fact that we observed deficits supports the notion that the anterior temporal-lobe plays a selective role in memory for unfamiliar faces (in young patients as it does in adults).

This observation addresses the question of neuronal plasticity and early brain damage (D. Zaidel, 1988). If other regions in the right hemisphere are specialized for facial processing, why did those regions not "take over" in RTLE patients? A reasonable answer would be that those other regions are specialized for facial processing functions that are different from those tapped in the present test. We may speculate that they normally serve special-purpose functions that cannot be disturbed by and reassigned to "takeover" by displaced anterior functions, regardless of how early the damage to anterior temporal-lobe regions has occurred.

It is worthwhile to discuss de Schonen's (1992) proposal that interhemispheric interaction is involved in facial processing. There may be some support for the proposal in the present results. The clue may lie in the sensitive delayed memory trial. With damage in left temporal regions, we observe an increased score on the delay trial, the reverse pattern of normal and right-sided damage. This increase is interpreted to reflect the intact functioning of the right hemisphere. This implies that, when the left hemisphere is damaged, an interhemispheric inhibition normally exerted by the left over the right is minimized, and consequently right hemisphere facial processing functioning is maximized. This argument assigns a role to the left hemisphere in facial processing. In adult splitbrain patients, the (relatively intact) left hemisphere was found to be highly competent in face identification (Levy, Trevarthen, \& Sperry, 1972). In the intact brain, there may be hemispheric interaction in facial processing even while the right is more competent than the left in this function; 
The forebrain commissures may be selectively involved in regulating facial processing. Similar arguments that the corpus callosum normally plays an inhibitory role during lateralized and unlateralized perceptual and cognitive tasks were made previously (e.g., Cooke, 1986; Lassonde, 1986; E. Zaidel, Clarke, \& Suyenobu, 1990).

From theoretical and clinical perspectives, the present preliminary findings illustrate the behavioral dynamics of early localized brain lesions. The behavioral consequences of the epilepsyrelated lesion have both reversible and irreversible components. Surgical or clinical management alone may prove insufficient in some cases and behavioral intervention may be useful so that compensatory strategies overcome the cognitive deficit. In the present group of patients, there was a continuum of deficit from immediate to delayed memory. However, even the relatively subtle deficits revealed in delayed memory may have far-reaching implications regarding facial processing in the complex social interaction of everyday life. Future studies of children suffering from early temporal-lobe epilepsy, or any other early brain damage, in which the sex of the patient is studied as well may provide additional important insights regarding the findings on epilepsy reported here.

Acknowledgments: This research was supported by NATO Collaborative Research Programme and by NIH Grant NS 20187. We thank Dr. J. M. Oxbury for permission to study his patients. We are grateful to the teachers and students in the Oxfordshire School District for participating in the study and, specifically, to L. Johns for assistance in collecting the normative data. We thank K. Carpenter, J. Taylor, and C. Passingham for help throughout the preparation of the manuscript. Special note: This paper was submitted for publication after the untimely death of the senior author, Elizabeth D. Beardsworth, a child neuropsychologist, who designed the study and collected the data. This paper is dedicated to her memory. 


\section{References}

Adams, C. B. T. (1987). Temporal lobectomy in young people. In D. C. E. Ross \& R. Crawford (Eds.), Epilepsy in young people (pp. 103-111). Chichester: Wiley.

Benton, A. L., \& Van Allen, M. W. (1968). Impairment in facial recognition in patients with cerebral disease. Cortex, $\underline{4}, 344-358$.

Benton, A. L., \& Van Allen, M. W. (1973). Test of facial recognition. (Neurosensory Center Publication No. 287) Iowa City: University Hospitals, Department of Neurology.

Campbell, R. (1992). Face to face: interpreting a case of developmental prosopagnosia. In R. Campbell (Eds.), Mental lives (pp. 216-236). Oxford: Blackwell.

Carey, S. (1981). The development of face perception. In G. Davies, H. Ellis, \& J. Shepherd (Eds.), Perceiving and remembering faces (pp. 11-38). London: Academic Press.

Carey, S. (1992). Becoming a face expert. Philosophical Transactions of the Royal Society, London B, $\underline{335}, 95-103$.

Carey, S., \& Diamond, R. (1977). From piecemeal to configurational representation of faces. $\underline{\text { Science, }} \underline{195}, 312-314$.

Carey, S., Diamond, R., \& Woods, B. (1980). The development of face recognition - a maturational component? Developmental Psychology, 16, 257-269.

Cooke, N. D. (1986). The brain code: Mechanisms of information transfer and the role of the Corpus Callosum (pp. 120-135). London: Methusen.

de Schonen, S. (1992). Discussion of 'Becoming a face expert'. Philosophical Transactions of the Royal Society London, B Series, $\underline{335}, 102$.

de Schonen, S., \& Mathivet, E. (1989). First come, first served: A scenario about the development of hemispheric specialization. European Bulletin of Cognitive Psychology, 9, 33-44.

DeRenzi, E., Faglioni, P. , \& Spinnler, H. (1968). The performance of patients with unilateral brain damage on face recognition tasks. Cortex, $\underline{5}, 274-284$. 
Ellis, H. D. (1990). Developmental trends in face recognition. The Psychologist: Bulletin of the British Psychological Society, $\underline{3}, 114-119$.

Ellis, H. D. (1992). The development of face processing skills. Philosophical Transactions of the $\underline{\text { Royal Society, London B, }}$ 335, 105-111.

Ellis, H. D., \& Flin, R. H. (1990). Encoding and storage effects in 7-year-olds' and 10-yearolds' memory for faces. British Journal of Developmental Psychology, $\underline{8}, 77-92$.

Lassonde, M. (1986). The facilitatory influence of the corpus callosum on intrahemispheric processing. In F. Lepore, M. Ptito, \& H. H. Jasper (Eds.), Two hemispheres - One brain: Functions of the corpus callosum (pp. 385-401). New York: Liss.

Levine, S. C. (1985). Developmental changes in right hemisphere involvement in face recognition. In C. T. Best (Ed.), Hemispheric Function and Collaboration in the Child (pp. 157-191).New York: Academic Press.

Levy, J.,Trevarthen, C., \& Sperry, R. W. (1972). Perception of bilateral chimeric figures following hemisphere deconnexion. Brain, $\underline{95}, 61-78$.

McConachie, H. R. (1977). Developmental prosopagnosia. A single case report. Cortex, $\underline{12}, 76-82$.

Milner, B. (1958). Psychological defects produced by temporal-lobe excision. Research Publication of the Association of Research in Nervous \& Mental Diseases, $\underline{36}, 244-257$. Milner, B. (1968). Visual recognition and recall after right temporal-lobe excision in man. Neuropsychologia, $\underline{6}, 191-209$.

Newcombe, F.,de Haan, E. H. F.,Ross, J., \& Young, A. W. (1989). Face processing, laterality and contrast sensitivity. Neuropsychologia, 27, 523-538.

Oxbury, J. M., \& Adams, C. B. T. (1989). Neurosurgery for epilepsy. British Journal of $\underline{\text { Hospital }}$ Medicine, $\underline{41}, 372-377$.

Oxbury, J. M., \& Oxbury, S. M. (1989). Neuropsychology: Memory and hippocampal pathology. In E. H. Reynolds \& M. R. Trimble (Eds.), The bridge between neurology and psychiatry (pp. 135-151). London: Churchill Livingstone. 
Rudel, R. G. (1985). Hemispheric asymmetry and learning disabilities: Left, right, or in-between. In C. T. Best (Ed.), Hemispheric function and collaboration in the child (pp. 275-308). New York: Academic Press.

Turkewitz, G., \& Ross-Kossak, P. (1984). Multiple modes of right hemisphere information processing: Age and sex differences in facial recognition. Development Psychology, $\underline{20}$, 95-103.

Tzavaras, A.,Hecaen, H., \& Le Bras, H. (1970). Le probleme de la specificite du deficit de las reconnaissance du visage humain lors des leions hemipheriques unilaterales. Neuropsychologia, $\underline{8}, 403-417$.

Warrington, E., \& James, M. (1967). An experimental investigation of facial recognition in patients with unilateral cerebral disease. Cortex, $\underline{3}, 317-326$.

Wechsler, D. (1974). Wechsler Intelligence Scale for Children - Revised. New York: The Psychological Corportion.

Yin, R. (1970). Face recognition by brain-injured patients: A dissociable ability. Neuropsychologia, $\underline{8}, 395-402$.

Zaidel, D. W. (1988). Observations on right hemisphere language functions. In C. F. Rose, R. Whurr, \& M. A. Wyke (Eds.), Aphasia (pp. 170-187). London: Whurr Publishers.

Zaidel, E., Clarke, J. M., \& Suyenobu, M. A. (1990). Hemispheric independence: A paradigm case in cognitive neuroscience. In A. F. Scheibel \& A. F. Wechsler (Eds.), Neurobiology of higher cognitive function (pp. 297-354). New York: Guilford. 


\section{FIGURE LEGENDS}

FIGURE 1: Mean accuracy scores (\%) of the normal group (average of Sets I and II) and of the LTLE and RTLE groups preoperatively.

FIGURE 2: Mean accuracy scores (\%) of the normal group (average of Sets I and II) and the patient groups postoperatively.

FIGURE 3: Performance of matched patients in the LTLE and the RTLE groups. They were administered the facial memory test both before and after surgery. 
Table 1

Means (Standard Error in Brackets) of Clinical Details and IQ Scores

\begin{tabular}{|c|c|c|}
\hline & \multicolumn{2}{|c|}{ Patient group } \\
\hline & Left $(\underline{n}=13)$ & $\operatorname{Right}(\underline{\mathrm{n}}=16)$ \\
\hline Age operated (yrs) & $14.0(.59)$ & $12.9(.59)$ \\
\hline \multicolumn{3}{|l|}{ Age of onset of } \\
\hline habitual epilepsy (yrs) & $5.1(.65)$ & $5.3(.65)$ \\
\hline \multirow[t]{2}{*}{ WISC-R Full Scale IQ } & pre-op $92.7(2.8)$ & pre-op $96.9(2.5)$ \\
\hline & post-op $93.2(2.8)$ & post-op 95.9 (2.9) \\
\hline \multirow[t]{2}{*}{ Verbal IQ } & pre-op $82.8(2.8)$ & pre-op $91.6(2.3)$ \\
\hline & post-op $82.6(3.5)$ & post-op $90.5(2.8)$ \\
\hline \multirow[t]{2}{*}{ Performance IQ } & pre-op $104.8(2.9)$ & pre-op $103.3(3.7)$ \\
\hline & post-op $107.6(3.1)$ & post-op $103.1(3.6)$ \\
\hline
\end{tabular}


Table 2

$\underline{\text { Intercorrelations between overall facial memory scores and epilepsy-related factors }}$

\section{Epilepsy factors}

Age at Age at History of Number of

Group surgeryonset epilepsy seizures

\begin{tabular}{lcccc} 
Left & & & \\
preoperative & $.72 *$ & .02 & .46 & -.27 \\
postoperative & .57 & .0 & -.07 & -.56 \\
& & & & \\
Right & & .38 & .26 & $.65^{*}$ \\
preoperative. & $79 *$ & .28 & .42 & $.71^{*}$ \\
postoperative & $.73^{*}$ & & & \\
\hline$* \mathrm{p}<.04$ & & &
\end{tabular}

